\title{
Development of technique for checking insulation distance for large-scale CAD data using voxel meshes
}

\author{
Takashi HAMAGUCHI*, Makoto ONODERA* and Takashi YOKOHARI** \\ *Hitachi, Ltd. Research \& Development Group, Center for Technology Innovation - Mechanical Engineering \\ 832-2, Horiguchi, Hitachinaka, Ibaraki 312-0034, Japan \\ E-mail: takashi.hamaguchi.zb@hitachi.com \\ ** Hitachi, Ltd. Research \& Development Group, Center for Technology Innovation - Materials \\ 7-1-1, Omika, Hitachi, Ibaraki 319-1292, Japan
}

Received: 27 June 2017; Revised: 2 April 2018; Accepted: 15 April 2018

\begin{abstract}
Conventionally, designers of electronic equipment structures visually inspect the insulation distance or check it by using CAD software functions. However, unintentionally failed detection and overdetection of objects being inspected can cause problems. Therefore, we are developing a technique for checking the insulation distance. The technique calculates the insulation distance by using voxels, which are in orthogonal meshes. The inputs of this technique involve checking conditions such as the clearance distance threshold and creepage distance threshold, setting part attributes such as of insulation and conduction, and calculating parameters such as voxel sizes. The output of this technique is the visualization of violating parts and paths detected by calculating the insulation distance and generating a distance map. We confirmed that our technique can measure the clearance and creepage distances, detect violation parts, and reduce overdetection of a model for inspection.
\end{abstract}

Keywords : Insulation distance, Computer aided design, Voxel mesh, Structural design, Electric equipment

\section{Introduction}

Electronic equipment needs to meet certain standards for insulation distance (IEC 60950-1, 2005)(JIS C 6950-1, 2012). The insulation distance is the distance between a high-potential part and a low-potential part. Two kinds of distance, clearance and creepage, are defined in the distance standard (Kobayashi et al., 2005). If the distance is less than a threshold, sparks may occur in the equipment. The insulation distances are different for each voltage and condition.

Conventionally, designers of electronic equipment structures visually inspect the insulation distance or check it by using CAD software functions. However, unintentionally failed detection and overdetection of objects being inspected can cause problems. For example, one function may overdetect pairs of a low-potential part and insulation part, pairs of a low-potential part and low-potential part, and pairs of a high-potential part and insulation part being obstructed by an insulation part.

The conventional methods for checking the insulation distance detect the shortest path and measure the distances between one high-potential part and one low-potential part. The shortest path and the distance of electrical creepage were researched by Kageura and Shimada et al. Conventional methods based on this research are intended for mainly twin parts. Therefore, measuring the insulation distance of a large number of part pairs will require many work hours.

The conventional methods for image processing calculate distance in a field. The methods are called "distance transforms" (Rosenfeld and Pfaltz, 1966). These methods were researched by Verwer, Grevera, Borgefors, Inui, Jones, Butt, Cuisenaire, Satherley, Marchand-Maillet et al. However, they may fail to detect violating parts for insulation distances because the calculated distance may be longer than the Euclidean distance.

Accordingly, to solve these problems, we are developing a technique for checking the insulation distance to calculate the distance by using voxels, which are in an orthogonal mesh. Our technique is for aiding in designing electronic equipment. The target is to conventionally reduce the number of work hours to half. 


\section{Technique for checking insulation distance \\ 2.1 Problems with conventional methods}

Insulation distances are shown in Fig. 1. If the distance is less than a threshold, sparks may occur in equipment. A summary of the conventional methods for checking insulation distances is shown in Table 1. Conventionally, designers of electronic equipment structures visually inspect the insulation distance or check it by using CAD software functions. However, unintentionally failed detection and overdetection of objects being inspected can cause problems. For example, one function may overdetect pairs of a low-potential part and insulation part, pairs of a low-potential part and lowpotential part, and pairs of a high-potential part and insulation part being obstructed by an insulation part, as shown in Figs. 2(a) and (b). The measurement distance is $\mathrm{L}_{\mathrm{m}}$, and the insulation distance threshold is $\mathrm{L}_{\mathrm{t}}$. Insulation distance thresholds are determined by selecting the voltage and condition.

As mentioned, the conventional methods for checking the insulation distance detect the shortest paths and measure the distances between high-potential parts and low-potential parts, and conventional methods are intended for mainly twin parts. Measuring the insulation distance of a large number of part pairs is time-consuming, so our technique for checking the insulation distance calculates the distance by using voxels in an orthogonal mesh. The target is to conventionally reduce the number of work hours to half.

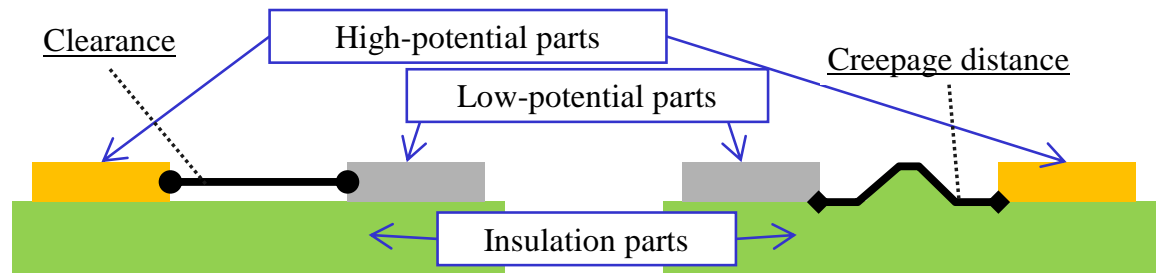

Fig. 1 Clearance and creepage distance

[The insulation distance is the distance between a high-potential part and low-potential part. Two kinds of distance, clearance and creepage, are defined in the distance standard. If the distance is less than a threshold, sparks may occur in equipment.]

Table 1 Summary of conventional methods for checking insulation distances

\begin{tabular}{|c|l|l|l|l|l|l|}
\hline \multirow{2}{*}{ No. } & \multicolumn{1}{|c|}{ Method } & \multicolumn{1}{|c|}{$\begin{array}{c}\text { Selection of } \\
\text { parts to verify }\end{array}$} & \multirow{2}{*}{$\begin{array}{l}\text { Detection } \\
\text { accuracy }\end{array}$} & $\begin{array}{c}\text { Creepage } \\
\text { distance } \\
\text { measurement }\end{array}$ & $\begin{array}{c}\text { Operation } \\
\text { time }\end{array}$ & Checking time \\
\hline 1 & $\begin{array}{l}\text { Visual inspection with } \\
\text { 3D-CAD }\end{array}$ & $\begin{array}{l}\text { User } \\
\text { selection }\end{array}$ & $\begin{array}{l}\text { Oversight of } \\
\text { selected parts }\end{array}$ & Possible & $\begin{array}{l}\text { Less than } \\
\text { one hour }\end{array}$ & Unnecessary \\
\hline 2 & $\begin{array}{l}\text { Clearance verification } \\
\text { function of 3D-CAD }\end{array}$ & $\begin{array}{l}\text { Automatic } \\
\text { (Full selection) }\end{array}$ & Overdetection & Impossible & $\begin{array}{l}\text { Several } \\
\text { minutes }\end{array}$ & Several hours \\
\hline 3 & $\begin{array}{l}\text { Method for finding } \\
\text { shortest path between } \\
\text { pair of points }\end{array}$ & $\begin{array}{l}\text { User } \\
\text { selection }\end{array}$ & $\begin{array}{l}\text { Oversight of } \\
\text { selected parts }\end{array}$ & Possible & $\begin{array}{l}\text { Less than } \\
\text { one hour }\end{array}$ & Unnecessary \\
\hline
\end{tabular}

Low-potential part Insulation part or low-potential part

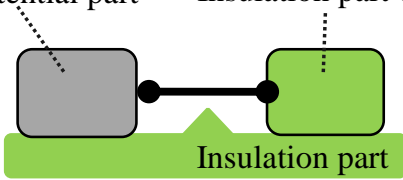

(a) Low-potential and insulation part

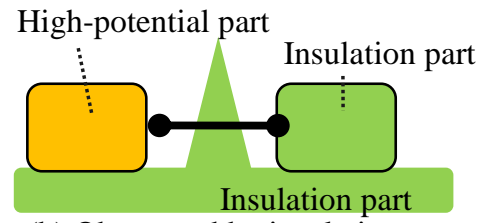

(b) Obstructed by insulation part

Fig. 2 Examples of overdetection

[Clearance verification function of 3D-CAD may overdetect pairs of a low-potential part and insulation part, pairs of a low-potential part and low-potential part, and pairs of a high-potential part and insulation part being obstructed by an insulation part.] 


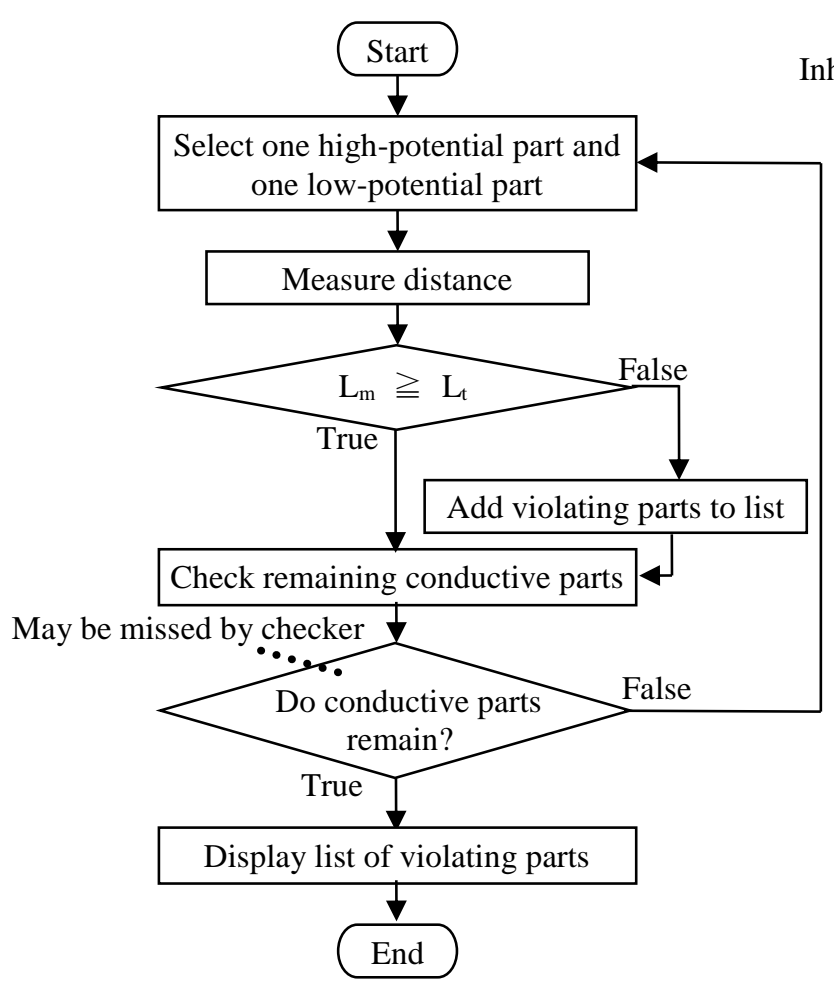

(a) Conventional method

(visual inspection, shortest path between pair of points)

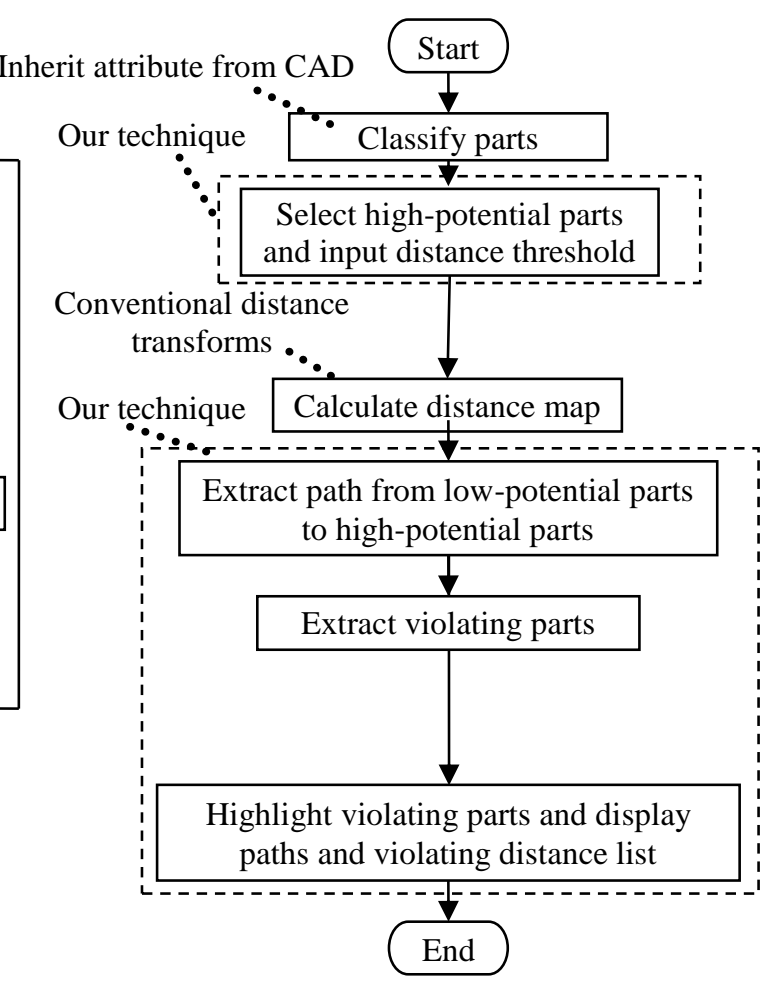

(b) Our technique

Fig. 3 Flow chart for checking of insulation distances

[Conventionally, designers of electronic equipment structures visually inspect the insulation distance. However, unintentionally failed detection of objects being inspected can cause problems.]

In Fig. 3, the conventional distance transforms are Chamfer distance transforms (Richard Satherley and Mark W. Jones, 2001) in Eq. (1) to solve the overdetection problem. However, these transforms may fail to detect violating parts for insulation distances because the calculated distance may be longer than the Euclidean distance.

$$
\begin{gathered}
\mathrm{D}(x, y, z)=\min \left(\left(D(x+i, y+j, z+k)+d_{\text {mat }}(i, j, k)\right) \forall i, j, k \in d_{\text {mat }}\right), \\
\text { where } \mathrm{x}, \mathrm{y}, \mathrm{z}, \mathrm{i}, \mathrm{j}, \mathrm{k} \in \mathbb{Z}
\end{gathered}
$$

With our technique, we extract paths from low-potential parts to high-potential parts. The paths are extracted on the basis of a map by using Chamfer distance transforms. Furthermore, the distances are re-calculated by subtracting an assumed maximum error. In Eqs. (2) and (3), the error is $\varphi$, the distance of the path is $\mathrm{L}_{\mathrm{p}}$, the Euclidean distance is $\mathrm{L}_{\mathrm{e}}$, and the calculated distance is $\mathrm{L}_{\mathrm{c}}$. Therefore, the calculated distance is shorter than the Euclidean distance.

$$
\begin{array}{r}
\varphi=L_{p}-L_{e} \\
L_{c}=L_{p}-\max (\varphi)
\end{array}
$$

\subsection{Overview of technique}

The inputs of our technique involve checking conditions such as the clearance distance threshold and creepage distance threshold, setting part attributes such as of insulation and conduction, and calculating parameters such as voxel sizes. An overview of this is shown in Fig. 4. Even complicated CAD models can be simplified to the voxels of an orthogonal mesh (Watanabe and Nakahashi, 2010). The calculation time will be short if simplified shapes are used. The output of this technique is the visualization of violating parts and paths detected by calculating the insulation distance and generating a distance map. The user's job is to mainly input parameters, such as thresholds, and to pick parts. The 
details on picking parts are given below.

The amount of work required to set part attributes is reduced by using a batch selection process for connecting parts, as shown in Fig. 5. First, users classify parts as conduction or insulation according to the material property in CAD. Next, they pick high-potential parts among conductive parts, and the associated parts are selected. Automatically, conductive parts connected to previously selected parts are searched for and selected as high-potential parts. This process iterates until no new parts are selected. By picking just a few parts, users can set part attributes easily.

The violating parts and paths are highlighted as shown in Fig. 6.

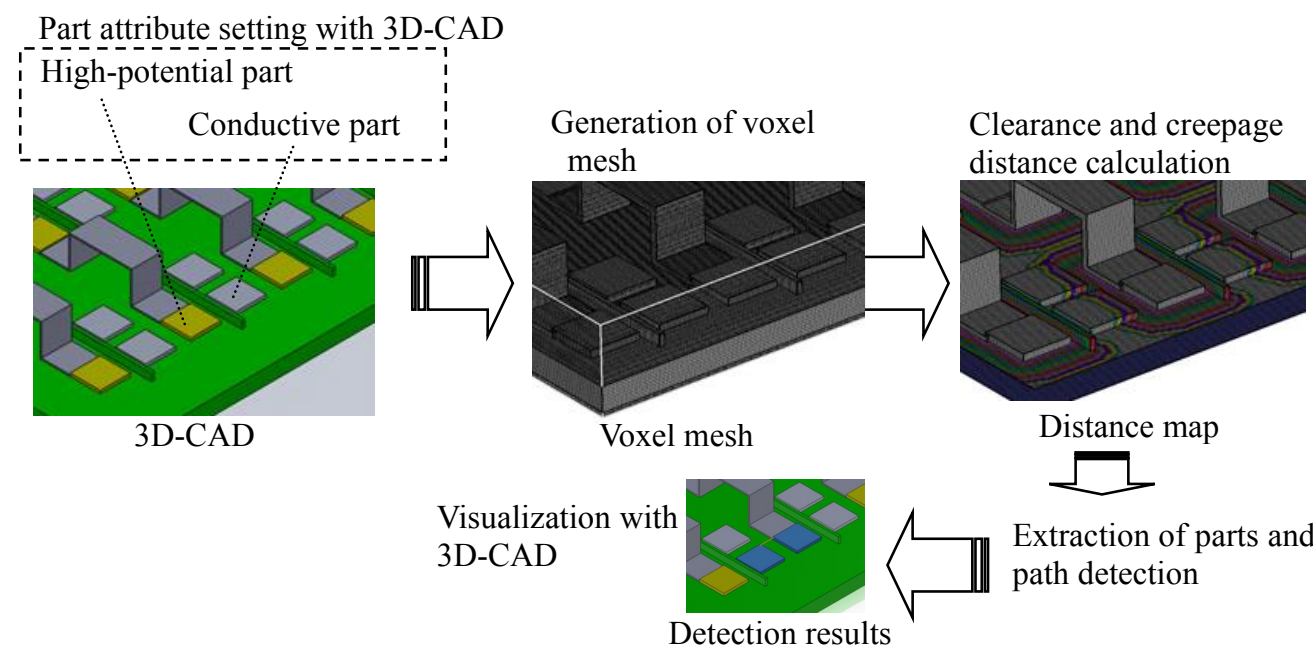

Fig. 4 Insulation check using voxel mesh

[Even complicated CAD models can be simplified to the voxels of an orthogonal mesh. The calculation time will be short if simplified shapes are used. The output of this technique is the visualization of violating parts and paths detected by calculating the insulation distance and generating a distance map.]

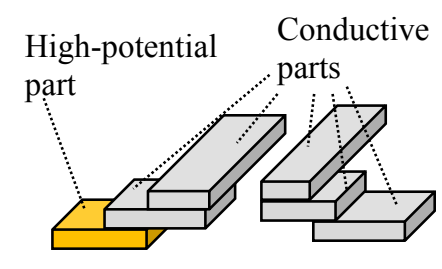

(a) Selecting high-potential parts

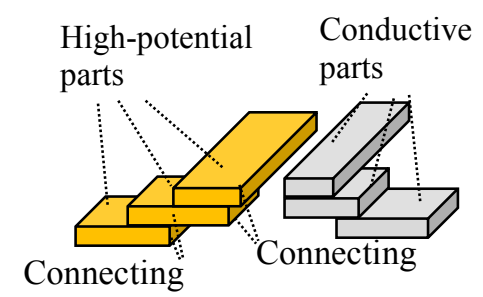

(b) Extracting high-potential parts

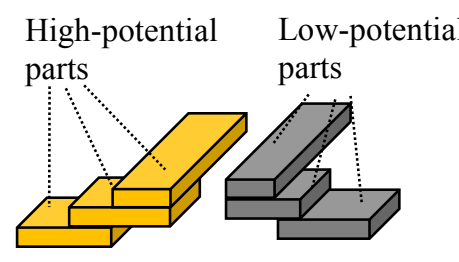

(c) Extracting low-potential parts

Fig. 5 Connected high-potential parts

[The amount of work required to set part attributes is reduced by using a batch selection process for connecting parts. First, users classify parts as conduction or insulation according to the material property in CAD. Next, they pick high-potential parts among conductive parts, and the associated parts are selected. Automatically, conductive parts connected to previously selected parts are searched for and selected as high-potential parts. This process iterates until no new parts are selected. By picking just a few parts, users can set part attributes easily.] 


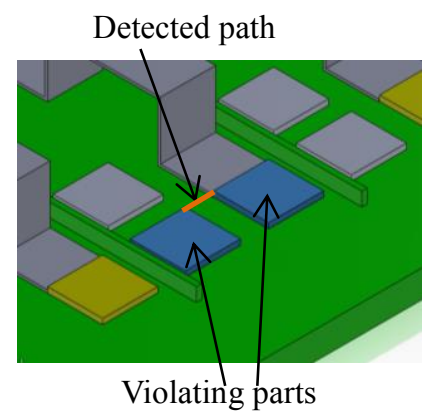

(a) Highlighted violating parts

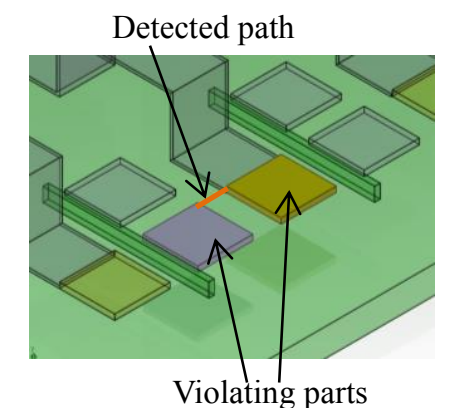

(b) Translucent body except violating parts

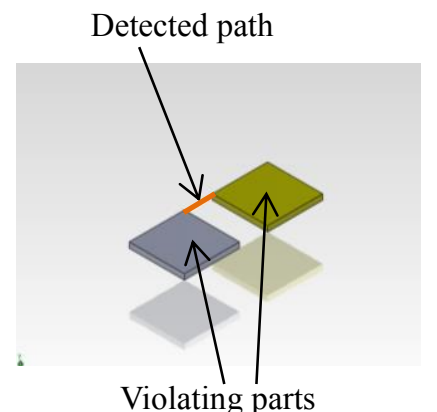

(c) Hidden body except violating parts

Fig. 6 Visualization of result

[The violating parts and paths are highlighted.]

\subsection{Technique for calculating to prevent failed detection}

Conventionally, distance transforms (DTs) (Rosenfeld and Pfaltz, 1966), known as distance field calculations, have been researched to measure the distance between cells of voxels in the image processing field. The Chamfer distance transform (CDTs) of an improved DT method could extract the shortest path approximately.

The clearance distance of our technique is calculated by using a quasi-Euclidean $5 \times 5 \times 5$ Chamfer distance matrix (Richard Satherley and Mark W. Jones, 2001). While the error of the distance is positive, the distance is longer than the Euclidean distance, as shown in Eq. (4). The error is $\varphi_{\mathrm{dt}}$, and distance of the path extracted by using CDTs is $\mathrm{L}_{\mathrm{pdt}}$. The Euclidean distance on the voxel mesh is $\mathrm{L}_{\mathrm{ev}}$. If the insulation distance is shorter than the threshold regulated by the electric standard, the risk of failed detection increases.

$$
\varphi_{d t}=L_{p d t}-L_{e v}
$$

Therefore, we calculate the distances of clearance and creepage on the basis of extracted paths, as shown in Fig. 7. In this case, the Chamfer distance is longer than the Euclidean one. Accordingly, the conventional method is inadequate for calculating the insulation distance. In contrast, distances calculated by our technique are shorter than the Euclidean distance. In the case of the clearance distance, the paths can cross insulation cells until the cell size is less than half. Furthermore, our technique subtracts the maximum $\varphi_{\mathrm{dt}}$ from the distance. The maximum $\varphi_{\mathrm{dt}}$ is shown in Eq. (5). The cell size is $\mathrm{L}_{\mathrm{vs}}$, the number of the corners of a path is $\mathrm{N}_{\mathrm{c}}$, and the coefficient is $\mathrm{C}$. The $\mathrm{C}$ of the clearance distance is the square root of 3 . The main error of the technique is the difference between the CAD shape and voxel mesh.

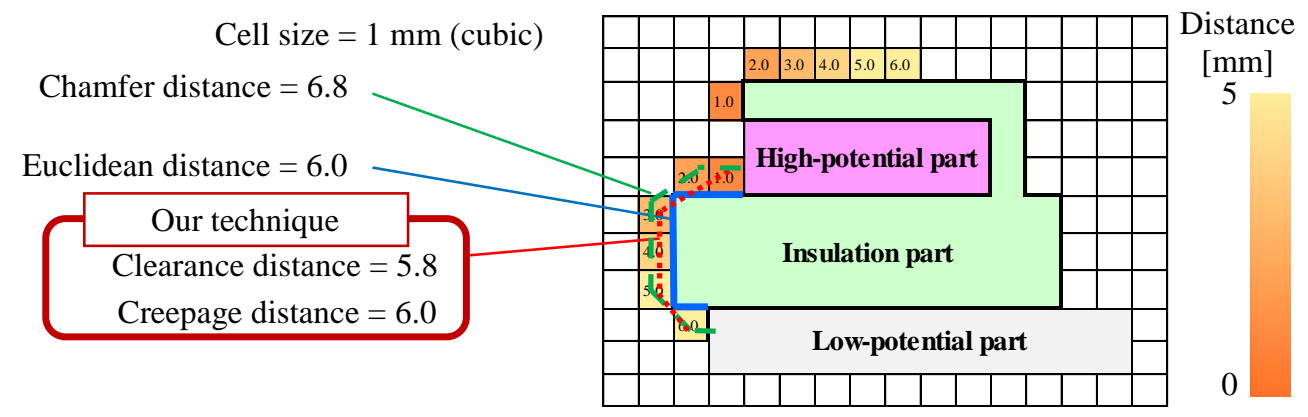

Fig. 7 Calculated insulation distance

[The distances of clearance and creepage are calculated on the basis of extracted paths.]

$$
\max \left(\varphi_{d t}\right)=L_{v s}\left(1+C N_{c}\right)
$$


The high-potential parts and low-potential parts of the voxel mesh of our technique expand in shape, as shown in Figs. 8 (a) and (b), and the shape of the insulation parts of the mesh contract, as shown in Fig. 8 (c). Therefore, the insulation distances on the voxel mesh are shorter than the CAD shape, as shown in Eq. (6). The Euclidean distance on the CAD shape is $\mathrm{L}_{\text {ecad. }}$.

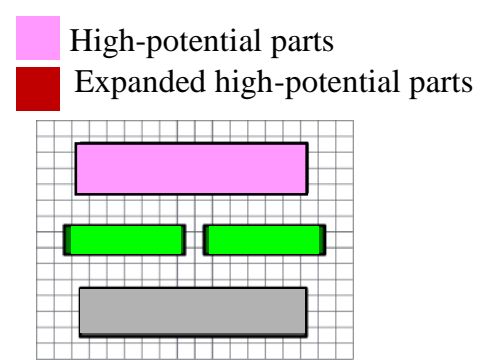

(a) CAD

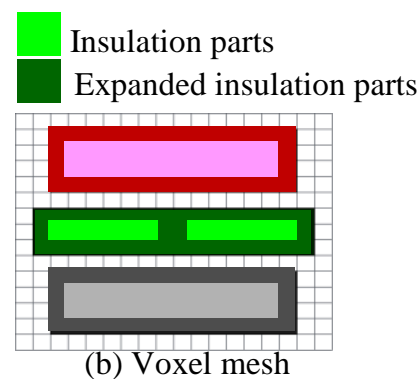

(Same method for all categories)
Low-potential parts

Expanded low-potential parts

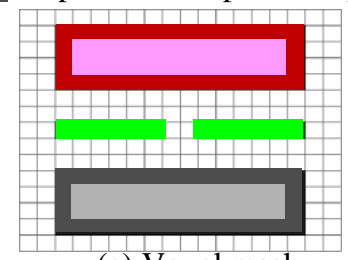

(c) Voxel mesh

(Our technique)

Fig. $8 \quad$ Voxel mesh for insulation check

[The high-potential parts and low-potential parts of the mesh of our technique expand in shape.]

$$
L_{\text {ecad }} \leq L_{e v}
$$

The distance map for the clearance distance is a quasi-Euclidean $5 \times 5 \times 5$ Chamfer distance matrix in our technique, as shown in Fig. 9.

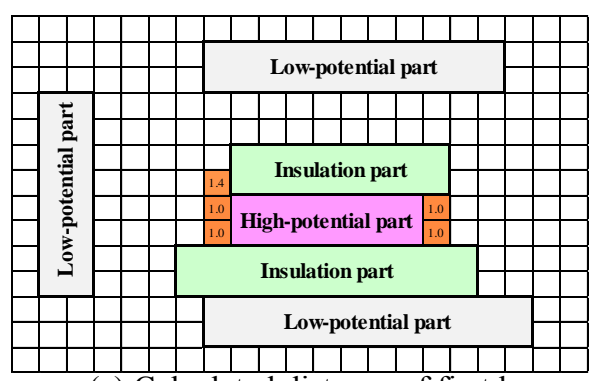

(a) Calculated distance of first layer

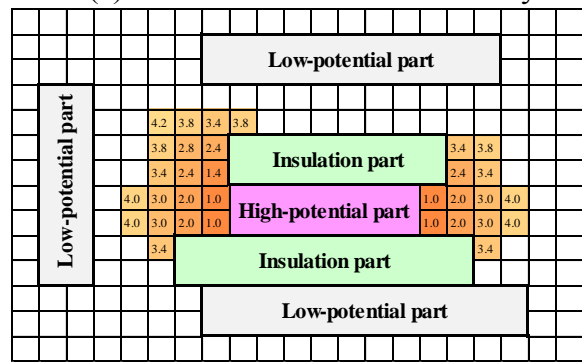

(c) Calculated distance of third layer

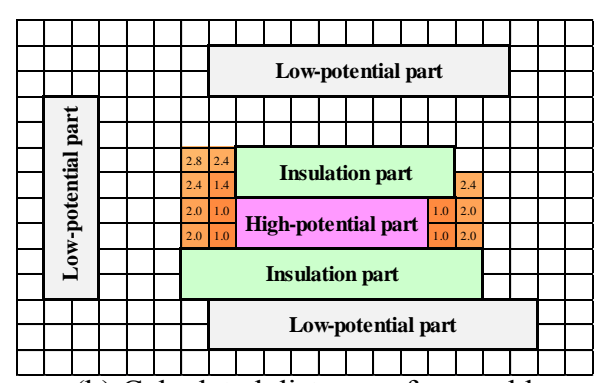

(b) Calculated distance of second layer

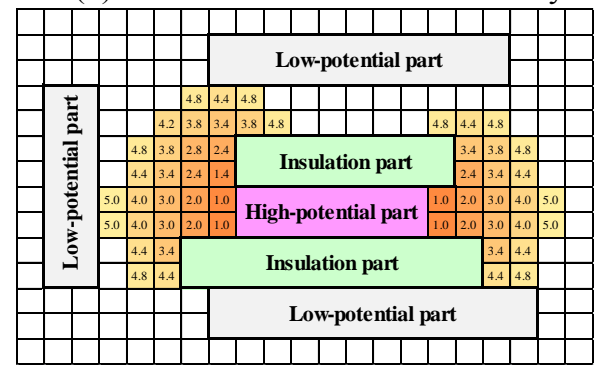

(d) Completed distance map

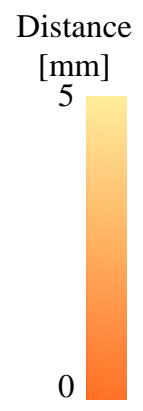

Fig. 9 Distance map for clearance distance

[The map for this distance is a quasi-Euclidean $5 \times 5 \times 5$ Chamfer distance matrix in our technique.]

Examples of distance values are shown in Fig. 10. Case 1 shows voxels from the side. Case 2 shows voxels diagonally in two dimensions. Case 3 shows voxels diagonally in three dimensions. 
Space cells for calculating distances

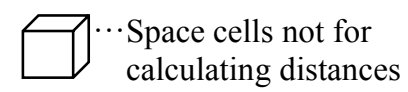

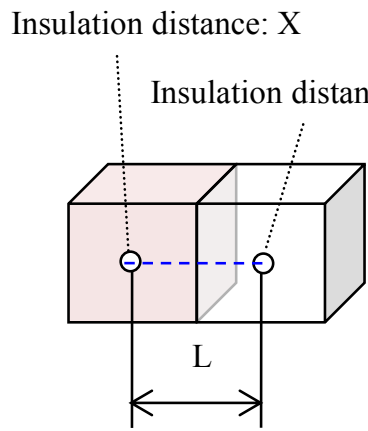

(a) Case 1
Insulation distance: $\mathrm{X}$

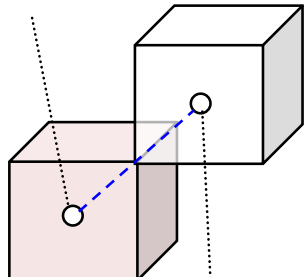

Insulation distance: $\mathrm{X}+\mathrm{L} \sqrt{2}$

(b) Case 2

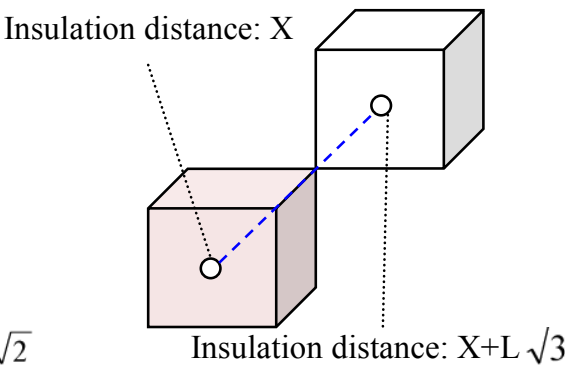

(c) Case 3

Fig. 10

Distance values for clearance distance

[Case 1 shows voxels from the side. Case 2 shows voxels diagonally in two dimensions. Case 3 shows voxels diagonally in three dimensions.]

The distance map for the creepage distance is a quasi-Euclidean $5 \times 5 \times 5$ Chamfer distance matrix in our technique, shown in Fig. 11.
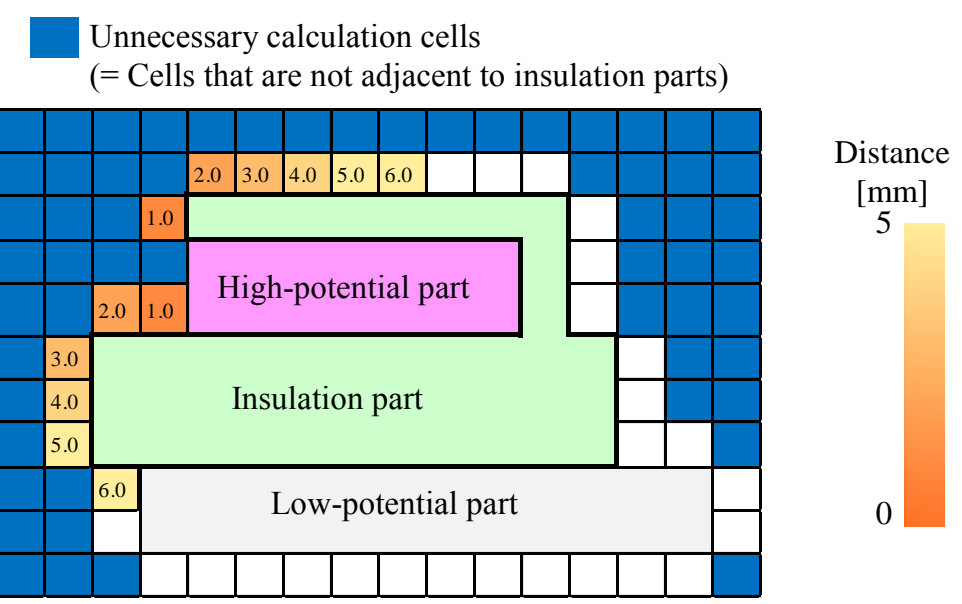

Fig. 11 Distance map for creepage distance

[The distance map for the creepage distance is a quasi-Euclidean $5 \times 5 \times 5$ Chamfer distance matrix in our technique.]

Examples of distance values are shown in Fig. 12. Case 1 shows voxels from the side. Cases 2 and 3 show voxels diagonally in two dimensions. Case 4 shows voxels diagonally in three dimensions. 


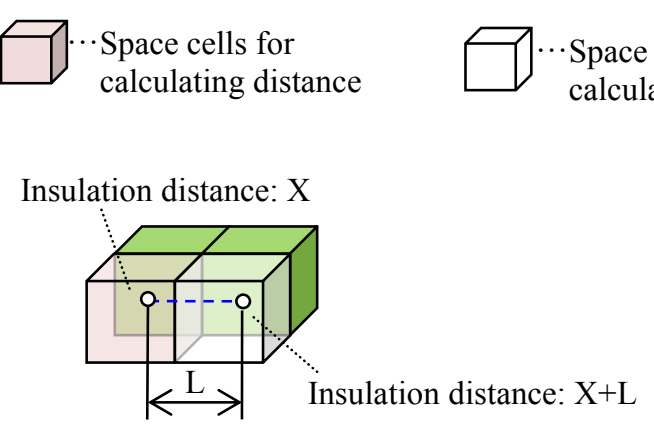

(a) Case 1

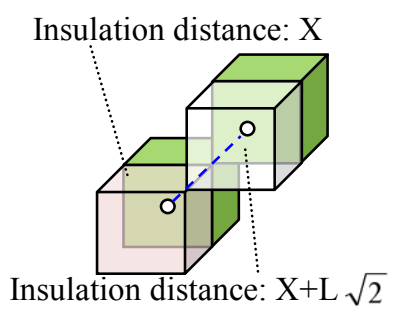

(c) Case 3
Space cells not for calculating distance $\cdot$ Insulation cells

\section{Insulation distance: $\mathrm{X}$}

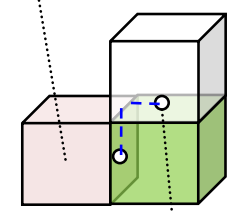

Insulation distance: $\mathrm{X}+\mathrm{L}$

(b) Case 2

Insulation distance: $\mathrm{X}$

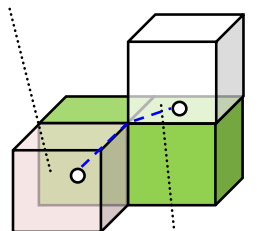

Insulation distance: $\mathrm{X}+\mathrm{L} \sqrt{2}$

(d) Case 4

Fig. 12 Distance value for creepage distance

[Case 1 shows voxels from the side. Cases 2 and 3 show voxels diagonally in two dimensions. Case 4 shows voxels diagonally in three dimensions.]

The start points of the path for extracting the clearance distance begin in the low-potential parts, as shown in Fig. 13 (a). The end points are in the high-potential parts. The path is a line that connects a cell whose value is added to the next cell value and whose distance to next cell is minimal, as shown in Figs. 13(b), (c), (d).

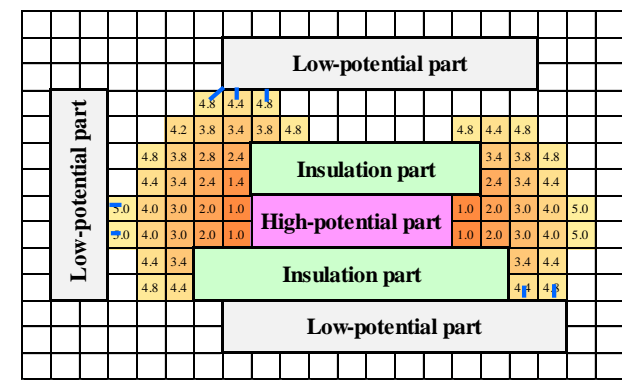

(a) First step

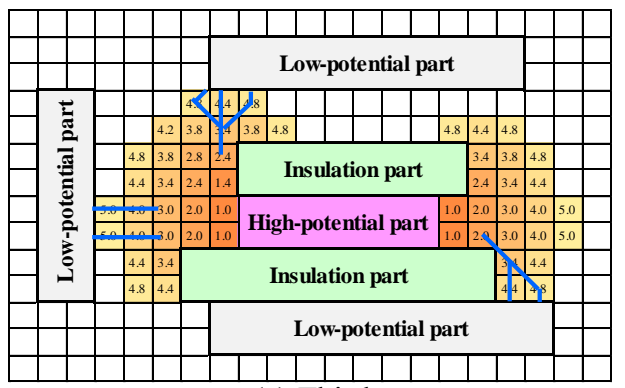

(c) Third step

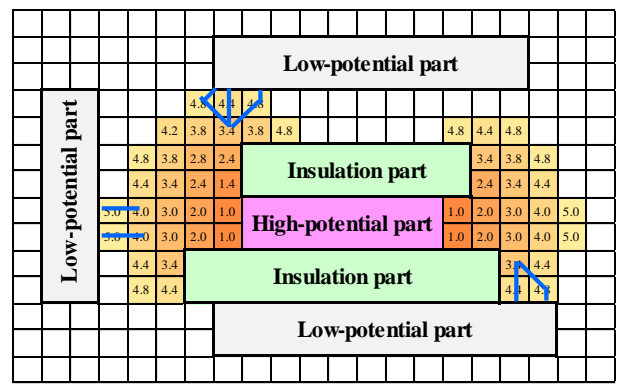

(b) Second step

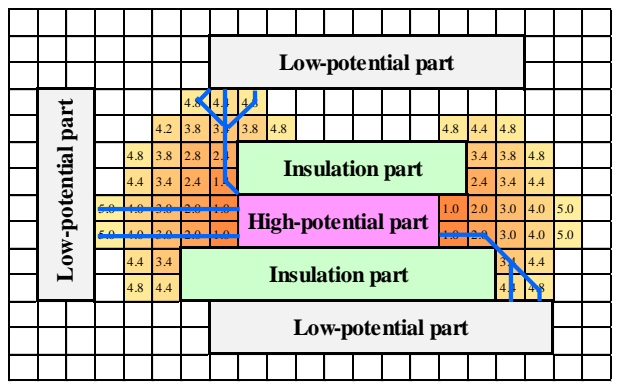

(d) Completed path

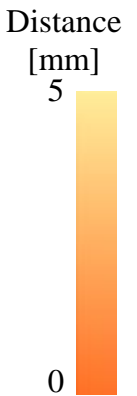

Fig. 13 Paths between high-potential and low-potential parts for clearance distance

[The start points of the path for extracting the clearance distance begin in the low-potential parts.] 
The start points of the path for extracting the creepage distance begin in the low-potential parts, and the end points are in the high-potential parts. The path is a line that connects a cell whose value is added to the face of the next cell value and whose distance to the face of the next cell is minimal, as shown in Fig.14. For the creepage distance, the C of Eq.

(5) is the square root of 2 .
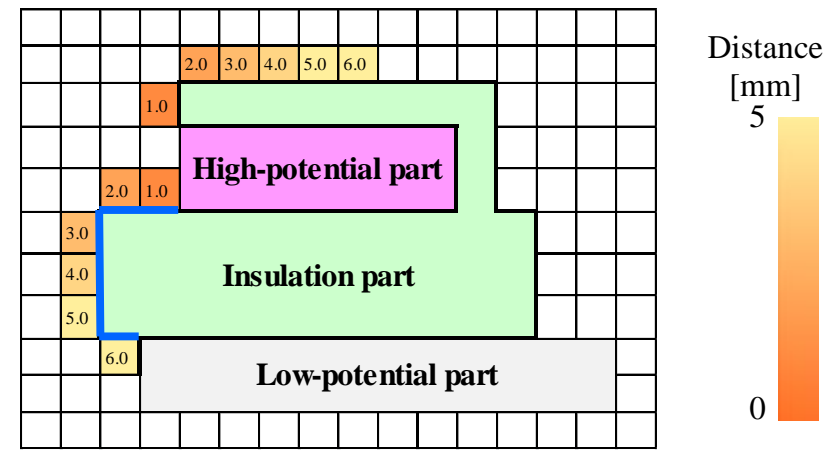

Fig. 14 Path between high-potential and low-potential parts for creepage distance

[The start points of the path for extracting the creepage distance begin in the low-potential parts, and the end points are in the high-potential parts. The path is a line that connects a cell whose value is added to the face of the next cell value and whose distance to the face of the next cell is minimal.]

\subsection{Verification of Technique}

The technique was verified, and it was found that all violating parts were detected by using test model 1, overdetections were fewer than 3D-CAD clearance verification with test model 2, work time was less than 3D-CAD clearance verification, and a large-scale 3D-CAD model can be checked by using test model 3 . The characteristics of the three test models are shown in Table 2.

Table 2 Characteristics of test model

\begin{tabular}{|l|l|l|l|}
\hline Model name & Human check & Shape & $\begin{array}{l}\text { Number of } \\
\text { conductive } \\
\text { parts }\end{array}$ \\
\hline Test model 1 & Practicable & Complex & 20 \\
\hline Test model 2 & Hard & Simple & 198 \\
\hline Test model 3 & Very hard & Simple & 10,029 \\
\hline
\end{tabular}

\section{Results}

\subsection{Test model 1}

To confirm that our technique can detect all violating parts, we checked the insulation distance of a test model, model 1, using the technique. The model was made up of four modules that had the same shape. The model is shown in Fig. 15. The span of the model was $102 \mathrm{~mm}$. Each of the modules had one high-potential part, four low-potential parts, and a few insulation parts. The clearance threshold of the model was $5 \mathrm{~mm}$, and the creepage distance threshold was $9 \mathrm{~mm}$, as shown in Table 3. The cell size of the calculating parameters was $0.015-0.2 \mathrm{~mm}$. The number of threads in parallel computing was four, equaling the number of modules. While the cell size was $0.015 \mathrm{~mm}$, the number of cells was $399,653,280$.

We confirmed that our technique can measure clearance and creepage distances and detect the violating parts of a model for inspection, as shown in Fig. 16.

The technique detected two low-potential clearance violating parts and four low-potential creepage distance violating parts. All of the detected parts were violating parts in the model. The results are shown in Fig. 17. The error was the absolute value obtained by subtracting the insulation distance calculated by using our technique from the actual distance determined by using CAD software functions. While the cell size was $0.015 \mathrm{~mm}$, the clearance maximum error was $0.7 \%$ $(0.03 \mathrm{~mm})$, and the creepage maximum error was $1.6 \%(0.11 \mathrm{~mm})$. The errors were sufficiently small considering that 
the span of the module was large. However, overdetection occurred in the error range. For example, if the cell size was $0.1 \mathrm{~mm}$ and $0.2 \mathrm{~mm}$, overdetection occurred. However, the bigger the cell size was, the shorter the calculation time was.

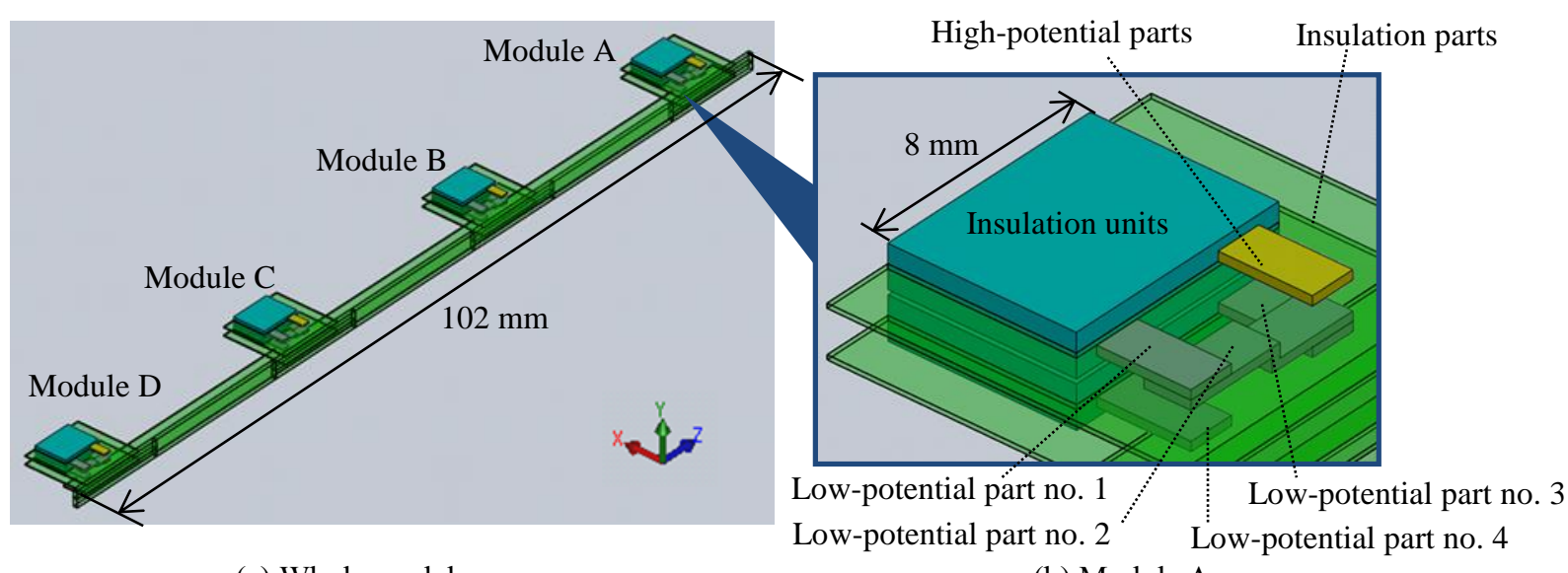

(a) Whole model

(b) Module A

Fig. 15 Test model 1 for missed detection

[To confirm that our technique can detect all violating parts, we checked the insulation distance of a test model, model 1, by using the technique. The model was made up of four modules that had the same shape.]

Table 3 Parameters of test model

\begin{tabular}{|c|c|c|c|}
\hline $\begin{array}{c}\text { Clearance } \\
\text { threshold } \\
{[\mathrm{mm}]}\end{array}$ & $\begin{array}{c}\text { Creepage distance } \\
\text { threshold } \\
{[\mathrm{mm}]}\end{array}$ & $\begin{array}{c}\text { Cell size } \\
{[\mathrm{mm}]}\end{array}$ & $\begin{array}{c}\text { Number of threads in } \\
\text { parallel computing }\end{array}$ \\
\hline 5 & 9 & $0.015-0.2$ & Max of 4 \\
\hline
\end{tabular}

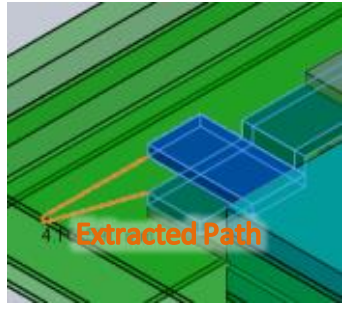

(a) Path of no. 2-C

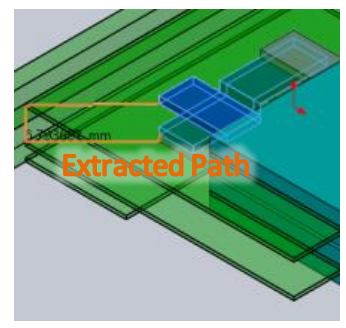

(d) Path of no. 2-CD

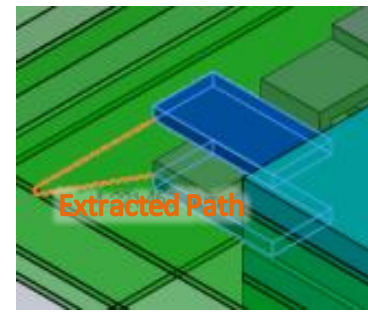

(b) Path of no. 3-C

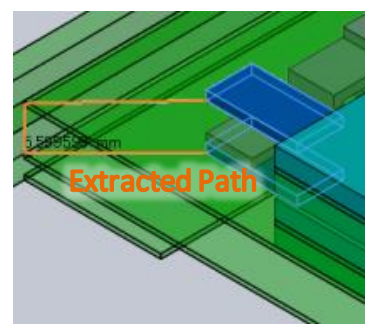

(e) Path of no. 3-CD

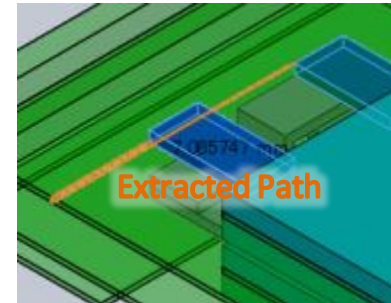

(c) Path of no. 1-CD

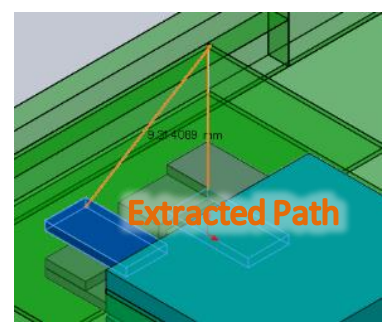

(f) Path of no. 4-CD

Distance type C: clearance CD: creepage distance

Fig.16 Extracted paths of low-potential parts

[Our technique can measure clearance and creepage distances and detect violating parts of a model for inspection.] 


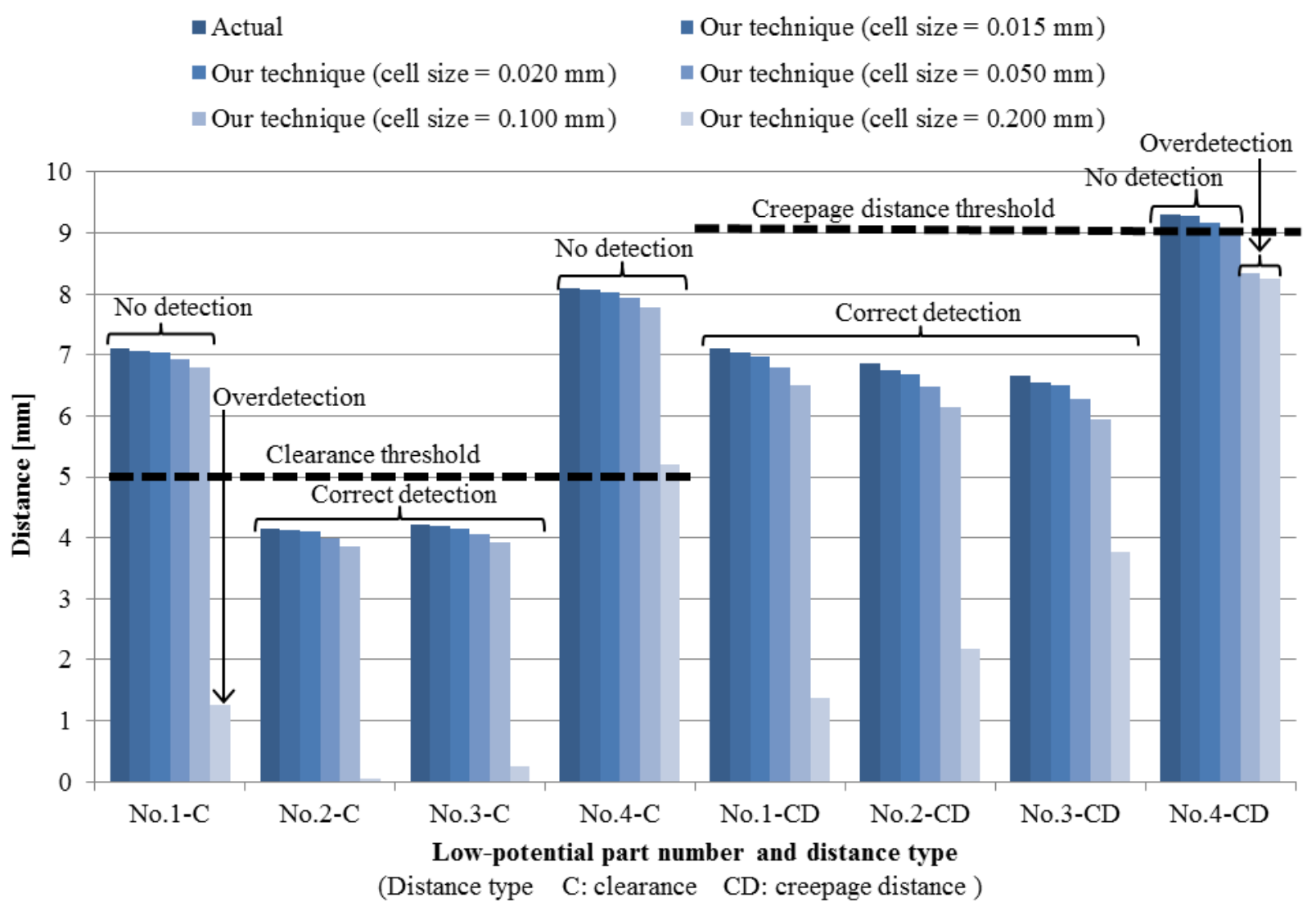

Fig. 17 Result of insulation distance measurements

[Overdetection occurred in the error range. For example, if the cell size was $0.1 \mathrm{~mm}$ and $0.2 \mathrm{~mm}$, an overdetection occurred.]

\subsection{Test model 2}

To determine the number of failed detections and overdetections, we used our technique to test model 2 and compared it with the conventional method (clearance verification). The model consisted of 99 high-potential parts, 99 lowpotential parts, and 46 insulation parts, as shown in Fig. 18 and Table 4. The clearance threshold of the model was 5 $\mathrm{mm}$, and the creepage distance threshold was $10 \mathrm{~mm}$. The cell size of the calculating parameters was $0.5 \mathrm{~mm}$. The number of cells was $12,571,200$.

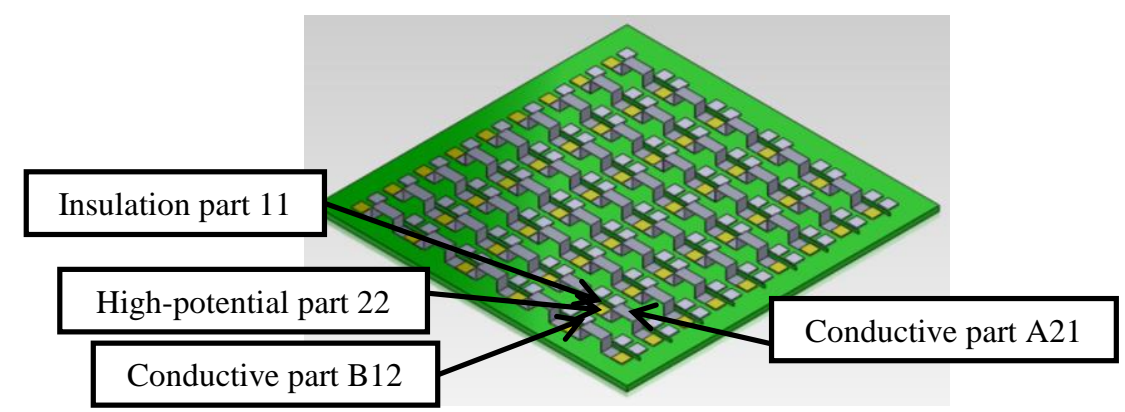

Fig. 18 Test model 2

[To confirm the number of failed detections and overdetections, we used our technique to test model 2 and compared it with the conventional method (clearance verification). The model consisted of 99 high-potential parts, 99 low-potential parts, and 46 insulation parts.] 
Table 4 Number of parts

\begin{tabular}{|c|c|}
\hline Category & Number of parts \\
\hline High-potential parts & 99 \\
\hline Low-potential parts & 99 \\
\hline Insulation parts & 46 \\
\hline Total & 244 \\
\hline
\end{tabular}

The work times of the model were $2.2 \mathrm{~min}$ with our technique and $5.2 \mathrm{~min}$ with the conventional method, as shown in Fig.19.

\section{- Selection of target parts $\square$ Distance calculation}

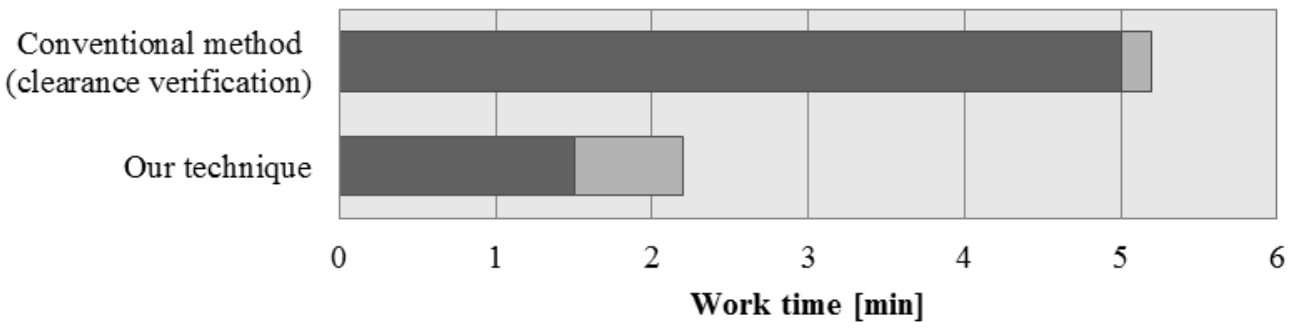

Fig. 19 Work time

[The work times of the model were $2.2 \mathrm{~min}$ with our technique and $5.2 \mathrm{~min}$ with the conventional method.]

The numbers of violating parts was 186 with our technique compared with 476 with the conventional method, as shown in Fig. 20. The numbers of overdetected parts were 298 with our technique compared with 8 with the conventional method. The numbers of missed detections were zero for both.

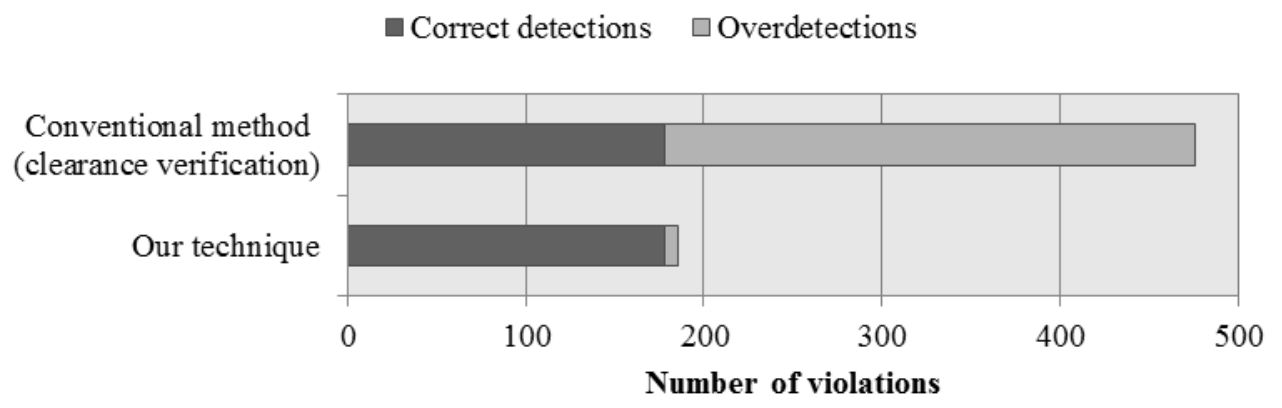

Fig. 20 Number of violations

[The numbers of violating parts was 186 with our technique compared with 476 with the conventional method. The numbers of overdetected parts were 298 with our technique compared with 8 with the conventional method. The numbers of missed detections were zero for both.]

\subsection{Test model 3}

To confirm the time taken to check the insulation distance of a large-scale model, we used our technique to test model 3 and compared it with the conventional method, as shown in Fig. 21. The model size was $500 \mathrm{~mm}$. The clearance threshold of the model was $1 \mathrm{~mm}$, and the creepage distance threshold was $2 \mathrm{~mm}$. The cell size of the calculating parameters was $0.5 \mathrm{~mm}$. The number of cells was 10,978,000.

The operation times were $5 \mathrm{~min}$ with our technique and $120 \mathrm{~min}$ with the conventional method, as shown in Fig. 22. The calculation times were $25 \mathrm{~min}$ with our technique and $20 \mathrm{~min}$ with the conventional method. The times taken to check the insulation distance were $30 \mathrm{~min}$ with our technique and $140 \mathrm{~min}$ with the conventional method. The number of work hours taken to check the insulation distance was reduced by $78 \%$ in the models. 


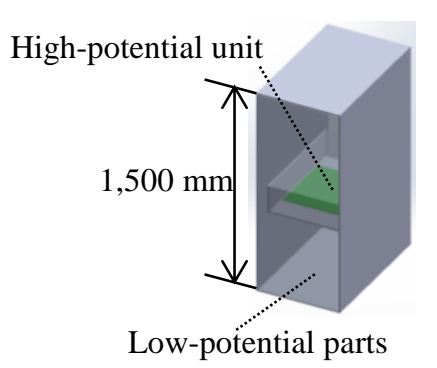

(a) Whole

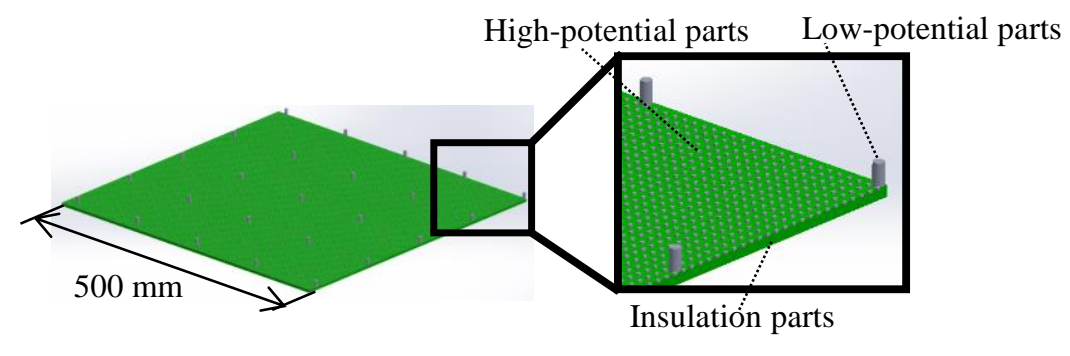

(b) Enlarged view of high-potential unit

Fig. 21 Large-scale model

[To confirm the time taken to check the insulation distance of a large-scale model, we used our technique to test model 3 and compared it with the conventional method.]

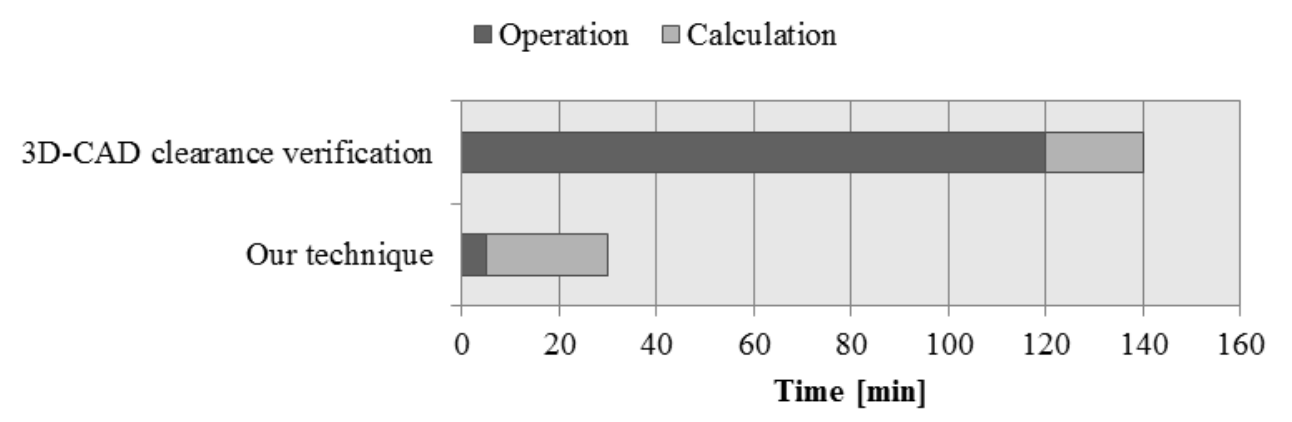

Fig. 22 Time for checking insulation distances

[The operation times were $5 \mathrm{~min}$ with our technique and $120 \mathrm{~min}$ with the conventional method. The calculation times were $25 \mathrm{~min}$ with our technique and $20 \mathrm{~min}$ with the conventional method. The times taken to check the insulation distance were $30 \mathrm{~min}$ with our technique and $140 \mathrm{~min}$ with the conventional method.]

\section{Discussion}

The errors were big so that the cell size was big, as Fig. 17 indicates. In low-potential part no. 1 of model 1, if the cell size was $0.1 \mathrm{~mm}$, the maximum error was $4.3 \%(0.31 \mathrm{~mm})$, and there was no overdetection. If the cell size was 0.2 $\mathrm{mm}$, the error was $82.2 \%(5.84 \mathrm{~mm})$, and overdetection occurred. Because insulation parts disappear due to the simplification caused by using voxels, the error for this cell size was too big. Accordingly, it is necessary for the cell size to be less than the minimum thickness of the insulation parts.

In model 2, which included 178 violating parts, the numbers of overdetected parts when using both the technique and conventional method were 8 and 298, respectively, when using CAD software functions. In model 3, the number of work hours using both was $5 \mathrm{~min}$ and $120 \mathrm{~min}$ when using CAD software functions. Then, the calculation times when using both were $25 \mathrm{~min}$ and $20 \mathrm{~min}$ when using CAD software functions. We confirmed that our technique reduces the number of overdetections and the number of work hours of a model for inspection. Accordingly, our technique will reduce the number of work hours to less than half.

\section{Conclusion}

Conventionally, designers of electronic equipment structures visually inspect the insulation distance or check it by using CAD software functions. However, unintentionally failed detection and overdetection of inspection objects can cause problems. Therefore, we are developing a technique for checking the insulation distance to calculate it by using voxels, which are in orthogonal meshes. The target is to conventionally reduce the number of work hours to half.

We applied our technique to test models and got the following conclusions. 
1. We confirmed that the technique could measure clearance and creepage distances and detect all violating parts of models for inspection.

2. We confirmed that the number of overdetected parts was eight (4\%) with our technique compared with 298 (167\%) with the conventional method when using CAD software functions for a model including 178 (100\%) violating parts.

3. We confirmed that the number of work hours taken to check the insulation distance was reduced by $78 \%$ in the models. Accordingly, our technique will reduce the number of work hours to less than half.

\section{References}

Borgefors, G., Distance Transforms in Arbitrary Dimensions, Computer Vision, Graphics, and Image Processing, Vol. 27, (1984), pp. 321-345.

Borgefors, G., Distance Transforms in Digital Images, Computer Vision, Graphics, and Image Processing, Vol. 34, (1986), pp. 344-371.

Borgefors, G., On Digital Distance Transforms in Three Dimensions, Computer Vision and Image Understanding, Vol. 64, No. 3 (1996), pp. 368-376.

Butt, M. A. and Maragos, P., Optimum Design of Chamfer Distance Transforms, IEEE Transactions on Image Processing, Vol. 7, No. 10 (1998), pp. 1477-1484.

Cuisenaire, O. and Macq, B., Applications of the Region Growing Euclidean Distance Transform: Anisotropy and Skeletons, Proceedings of International Conference on Image Processing, Vol. 1 (1997), pp. 200-203.

Cuisenaire, O., Distance Transformations: Fast Algorithms and Applications to Medical Image Processing, PhD thesis. Université Catholique de Louvian (1999).

Dijkstra, E. W., A Note on Two Problems in Connexion with Graphs, Numerische Mathmatik 1 (1959), pp. 269-271.

Grevera, G. J., Distance Transform Algorithms and Their Implementation and Evaluation, Deformable Models Biomedical and Clinical Applications (2007), pp. 33-60.

Hamaguchi, T., Onodera, M. and Yokohari, T., Development of Insulation Distance Check Technique for Large-scale CAD Data, Proceedings of the 25th Design \& Systems Conference (2015)(in Japanese).

Hamaguchi, T. and Onodera, M., Development of Insulation Distance Check Technique Using 3D Shape Data, Proceedings of the 24th Design \& Systems Conference (2014)(in Japanese).

IEC 60950-1:2005, Information Technology Equipment - Safety - Part 1: General Requirements, International Electrotechnical Commission (2005).

Inui, M., Umezu, N., Wakasaki, K. and Sato, S., Thickness and Clearance Visualization Based on Distance Field of 3D Objects, Journal of Computational Design and Engineering 2 (2015), pp. 183-194.

JIS C 6950-1:2012, Information Technology Equipment - Safety - Part 1: General Requirements, Japanese Standards Association (2012) (in Japanese).

Jones, M. W., Bærentzen, J. A. and Sramek, M., 3D Distance Fields: A Survay of Techniques and Applications, IEEE Transactions on Visualization and Computer Graphics, Vol. 12 No. 4 (2006), pp. 581-599.

Kageura, M. and Shimada, K., Finding the Shortest Path on a Polyhedral Surface and Its Application to Quality Assurance of Electric Components, Transactions of the ASME, Journal of Mechanical Design, Vol. 126 (2004), pp. $1017-1026$.

Kobayashi, Y., Insulation Distance Tester of the Three-dimensional Digital Product Model, Japanese Patent Disclosure 2005-18664 (2005)(in Japanese).

Marchand-Maillet, S. and Sharaiha, Y. M., Euclidean Ordering via Chamfer Distance Calculations, Computer Vision and Image Understanding, Vol. 73, Issue 3 (1999), pp. 404-413.

Rosenfeld, A. and Pfaltz, J. L., Sequential Operations in Digital Picture Processing, J. ACM, 13(4), (1966), $471-494$.

Satherley, R. and Jones, M. W., Vector-City Vector Distance Transform, Computer Vision and Image Understanding 82 (2001), pp. 238-254.

Verwer, B. J. H., Verbeek, P. W. and Dekker, S. T., An Efficient Uniform Cost Algorithm Applied to Distance Transforms, IEEE Transactions on Pattern Analysis and Machine Intelligence, Vol. 11, No. 4 (1989), pp. $425-429$.

Watanabe, M. and Nakahashi, K., Incompressible Flow Computations by the Modified Building-Cube Method, Transactions of the Japan Society of Mechanical Engineers, Series B, Vol. 76, No. 772 (2010), pp. 2018-2023 (in Japanese). 